J. Austral. Math. Soc. 22 (Series A) (1976), 1-11.

\title{
CONSECUTIVE INTEGERS WITH NO LARGE PRIME FACTORS
}

\author{
Dedicated to George Szekeres on his 65th Birthday \\ R. B. EGGLETON and J. L. SELFRIDGE
}

(Received 18 March 1975)

Communicated by Jennifer Seberry Wallis

\begin{abstract}
For fixed integers $k$ and $m$, with $k \geqq m \geqq 2$, there are only finitely many runs of $m$ consecutive integers with no prime factor exceeding $k$. We obtain lower bounds for the last such run. Let $g(k, m)$ be its smallest member. For $2 \leqq m \leqq 5$ it is shown that $g(k, m)>k^{\text {c logese }}$ holds for all sufficiently large $k$, where $c$ is a constant depending only on $m$. We also obtain a number of lower bounds with explicit ranges of validity. A typical result of this type is that $g(k, 3)>k^{3}$ holds just if $k \geqq 41$.
\end{abstract}

\section{Introduction}

For integers $k \geqq m \geqq 2$, let $g(k, m)$ denote the least integer such that any $m$ consecutive integers greater than $g(k, m)$ include a multiple of some prime exceeding $k$. In particular $g(k, 2)$ and $g(k, 2)+1$ are the largest consecutive integers with no prime factor greater than $k$. (For example, it is simple to show that $g(3,2)=8$.)

The function $g$ was introduced by Ecklund and Eggleton (1972), following the work of Erdös (1955) and Lehmer (1964, 1965). Recent numerical results have been reported by Ecklund, Eggleton and Selfridge (1973), and others are forthcoming.

The existence of $g(k, 2)$ follows from a theorem of Størmer (1897). Existence of $g(k, m)$ for $k \geqq m \geqq 2$ follows, since $g(k, m+1)<g(k, m)$ is immediate from the definition of $g$. Because of this monotonicity, $m=2$ is the most important case to study. Ecklund and Eggleton (1972) report that Erdös

The research of the first author has been supported by the Foundation for Number Theory Computing. 
suggested that perhaps $g(k, 2) \sim \exp \sqrt{k}$, but we seem to be far from proving or disproving this. An upper bound is given by the results of Lehmer (1964), which imply that

$$
\log g(k, 2)<c k^{2} \exp (k / 2)
$$

for some positive constant $c$. The lower bound

$$
g(k, 2)>k^{2} \quad \text { just if } k \geqq 5
$$

was proved by Ecklund and Eggleton (1972) by slightly sharpening an argument of Erdös.

In this paper we shall establish several lower bounds for $g(k, m)$ with $m=2,3,4,5$. The idea of using neighbours to a perfect power, which we employ in Section 2 is due to A. Schinzel and was communicated to us by $P$. Erdös. Next we employ solutions to the Prouhet-Tarry-Escott problem, in Section 3, and finally combine both methods in numerical work discussed in Section 4.

\section{Neighbours of a perfect power}

Sets of consecutive integers with no relatively large prime factors can be constructed by making use of the factorization

$$
z^{n}-1=\prod_{d \mid n} \Phi_{d}(z)
$$

where $\Phi_{d}$ is the cyclotomic polynomial whose roots are the primitive $d$ th roots of unity, and the product is taken over all positive divisors $d$ of $n$. The degree of $\Phi_{d}$ is $\phi(d)$, where $\phi$ is Euler's totient function. If $z_{0}$ is any complex number on the unit circle, $\left|z-z_{0}\right| \leqq|z|+1$ so

$$
\left|\Phi_{d}(z)\right| \leqq(|z|+1)^{\phi(d)} .
$$

We are now ready to prove

THEOREM 1. For any positive integer $n$, and $2 \leqq m \leqq 5$,

$$
g(k, m)>k^{n}
$$

for all sufficiently large integers $k$.

Proof. By (3) we have

$$
\left(2^{r}-1\right)\left(2^{r}+1\right)=2^{2 r}-1=\prod_{d \mid 2 r} \Phi_{d}(2)
$$

and

$$
\left(2^{r}-2\right)\left(2^{r}+2\right)=4\left(2^{2 r-2}-1\right)=4 \prod_{d \mid 2 r-2} \Phi_{d}(2) .
$$


Hence (4) shows that none of the five consecutive integers $2^{r}-2,2^{r}-1,2^{r}$, $2^{r}+1, \quad 2^{r}+2$ has a prime factor greater than $3^{\delta}$, where $\delta=$ $\max \{\phi(2 r), \phi(2 r-2)\}$.

Now we choose $r$ so that $\delta / r$ is small. For any positive integer $n$, the divergence of the product $I I(1-1 / p)$, taken over all primes, ensures that there exist positive integers $P_{0}$ and $P_{1}$ which are squarefree and coprime, and have enough prime factors to satisfy

$$
\prod_{p \mid P_{i}}\left(1-\frac{1}{p}\right)<\frac{1}{4 n}, \quad i=0,1 .
$$

By the Chinese Remainder Theorem there are infinitely many positive integers $r$ such that simultaneously $r \equiv 0\left(\bmod P_{0}\right)$ and $r \equiv 1\left(\bmod P_{1}\right)$. For any such $r$, (6) implies

$$
\frac{\phi(2 r)}{2 r}=\prod_{p \mid 2 r}\left(1-\frac{1}{p}\right) \leqq \prod_{p \mid P_{0}}\left(1-\frac{1}{p}\right)<\frac{1}{4 n},
$$

and

$$
\frac{\phi(2 r-2)}{2 r}<\frac{\phi(2 r-2)}{2 r-2}=\prod_{p \mid 2 r-2}\left(1-\frac{1}{p}\right) \leqq \prod_{p \mid P_{1}}\left(1-\frac{1}{p}\right)<\frac{1}{4 n} .
$$

Thus $\delta<r / 2 n$ by (7) and (8), so for any $k$ satisfying $3^{r} \leqq k^{2 n}<3^{r+P_{0} P_{1}}$ and all sufficiently large $r$, we have $g(k, 5) \geqq g\left(3^{\delta}, 5\right) \geqq 2^{r}-2>3^{\left(r+P_{0} P_{1}\right) / 2}>k^{n}$. Thus the case $m=5$ of (5) holds for all sufficiently large $k$. The cases $m=2,3,4$ follow immediately.

A refinement of this argument allows us to replace $n$ by a slowly increasing function of $k$, as follows. Let $\left(s_{n}\right)_{n \geqq 1}$ and $\left(\alpha_{n}\right)_{n \geqslant 1}$ be sequences of positive integers in which $s_{n}$ is the product of the first $n$ primes and $2^{\alpha_{n}}<p_{n+1}<2^{\alpha_{n}+1}$, where $p_{n+1}$ is the $(n+1)$ st prime. If $r=2^{\beta} s_{n}$, with $0 \leqq \beta \leqq \alpha_{n}$, the two consecutive integers $2^{r}-1$ and $2^{r}$ have no prime factors greater than $3^{\phi(r)}$. For any $k$ satisfying $3^{\phi(r)} \leqq k<3^{\phi(2 r)}$ we have $g(k, 2) \geqq 2^{r}-1$. Now $I_{i \leq n}\left(1-1 / p_{i}\right) \sim$ $e^{-\gamma} / \log p_{n}$, where $\gamma$ is Euler's constant. Thus $g(k, 2)>k^{c \log p_{n}}$ for some positive constant $c$ and all sufficiently large $n$. (For example, our argument would hold with $c=1 / 2$.) Finally, $\log s_{n} \sim p_{n}$ leads to the result: $g(k, 2)>k^{c \log \log \log k}$ for all sufficiently large $k$. Evidently the argument extends to the cases $m=3,4,5$ so we have

THEOREM 2. For all sufficiently large integers $k$, and $2 \leqq m \leqq 5$,

$$
g(k, m)>k^{\text {clogloglogk }},
$$

where $c$ is a positive constant depending only on $m$.

The neighbours of a perfect power will be used again in $\$ 4$ to derive some 
lower bounds with explicit ranges of validity. Incidentally, it should be noted that the proofs of Theorems 1 and 2 are effective: numerical bounds on $k$ can be determined, and for any appropriate set of parameters (including $k$ ) a corresponding $m$-tuple can be constructed.

\section{Prouhet-Tarry-Escott solutions}

We now give another construction for sets of consecutive integers with no relatively large prime factors, based on solutions of the Prouhet-Tarry-Escott problem. This is the problem of determining a nontrivial integer sequence $\left(a_{i}\right)_{i=1}^{2 n}$, for some $n \geqq 2$ (called the order) such that

$$
\sum_{i=1}^{n} a_{i}^{r}=\sum_{i=7}^{n} a_{n+i}^{r} \quad \text { for } \quad r=1,2, \cdots, n-1 .
$$

Without loss of generality we may also require that $\prod_{i=1}^{n} a_{i}<\prod_{i=1}^{n} a_{n+i}$. Hardy and Wright (1960) report that (10) has solutions at least for $n \leqq 10$. Because of the relatively incomplete state of knowledge concerning Prouhet-Tarry-Escott solutions, we cannot determine the asymptotic order of the lower bound which our construction implies. However, the theoretical interest of the construction remains, and in $\$ 4$ it will be seen to be useful also for deriving certain lower bounds with explicit ranges of validity.

Let $A, B$ be polynomials whose roots are a solution to (10):

$$
A(x)=\prod_{i=1}^{n}\left(x-a_{i}\right)
$$

and

$$
B(x)=\prod_{i=1}^{n}\left(x-a_{n+i}\right) .
$$

Then $B(x)-A(x)=d$, where $d=\prod_{i=1}^{n} a_{n+i}-\prod_{i=1}^{n} a_{i}$. There are infinitely many integers $t>\mu$ such that $A(t), B(t)$ are positive multiples of $d$, and then $A(t) / d, B(t) / d$ are consecutive positive integers with no prime factor larger than $t-\lambda$, where $\lambda=\min \left\{a_{i}: 1 \leqq i \leqq 2 n\right\}$ and $\mu=\max \left\{a_{i}: 1 \leqq i \leqq 2 n\right\}$. Thus $g(k, 2) \geqq A(t) / d$, provided $k \geqq t-\lambda$.

Let $\nu=\max \{d-\mu+\lambda, \alpha\}$, where $\alpha$ is the greatest difference between consecutive members of $\left\{a_{i}: 1 \leqq i \leqq 2 n\right\}$ when arranged in increasing order of magnitude. The construction described above leads to an effective proof of

TheоRem 3. Suppose the Prouhet-Tarry-Escott problem has a solution of order $n$. If the parameters of the solution are $d, \lambda, \mu, \nu$, then

$$
g(k, 2)>k^{n \xi} / d,
$$

for all sufficiently large integers $k$, where $\xi=1+1 /(\mu-\lambda+\nu)$. 
Proof. Take a solution $\left(a_{i}\right)_{i=1}^{2 n}$ to (10), with parameters $d, \lambda, \mu, \nu$. Suppose $S$ is any set of $\mu-\lambda+\nu$ consecutive integers, none of which has a prime factor greater than $k$. Let $S_{1}=\left\{t-a_{i}: 1 \leqq i \leqq 2 n\right\}$. There are $\nu$ district choices of $t$ such that $S_{t} \subseteq S$. Now $|S|=\mu-\lambda+\nu \geqq d$, by definition of $\nu$, so $S$ contains at least one multiple of $d$. Since $\nu \geqq \alpha$, every member of $S$ belongs to some set $S_{t} \subseteq S$, so there is a $t_{0}$ such that $S_{t_{0}} \subseteq S$ and $S_{t_{0}}$ contains a multiple of $d$.

For any $k \geqq \mu-\lambda+\nu$, the smallest member of $S$ can be taken to be $g(k, \mu-\lambda+\nu)$. With $t_{0}$ chosen appropriately, the consecutive integers $A\left(t_{0}\right) / d$, $B\left(t_{0}\right) / d$ justify the conclusion that

$$
g(k, 2) \geqq A\left(t_{0}\right) / d>g(k, \mu-\lambda+\nu)^{n} / d .
$$

Ecklund and Eggleton (1972) effectively show that for any $\varepsilon>0$,

$$
\log g(k, m)>\exp \left(\frac{1}{m}-\varepsilon\right) \log k
$$

for all sufficiently large $k$. Hence $g(k, m)>k^{1+1 / m}$ holds for all sufficiently large $k$, and now the theorem follows from (12).

The method just used can also be applied to establish a lower bound on $g(k, 3)$. Let $\left(a_{i}\right)_{i=1}^{6}$ be a nontrivial sequence of integers such that

$$
a_{1}+a_{2}=a_{3}+a_{4}=a_{5}+a_{6}
$$

and

$$
a_{1} a_{2}+a_{5} a_{6}=2 a_{3} a_{4} .
$$

Without loss of generality, we may require $a_{1} a_{2}<a_{3} a_{4}<a_{5} a_{6}$. These three products are in arithmetic progression, by (14); let $d$ be the common difference. Let $\quad P_{1}(x)=\left(x-a_{1}\right)\left(x-a_{2}\right), \quad P_{2}(x)=\left(x-a_{3}\right)\left(x-a_{4}\right), \quad$ and $\quad P_{3}(x)=$ $\left(x-a_{5}\right)\left(x-a_{6}\right)$. There are infinitely many integers $t$ such that $P_{1}(t) / d, P_{2}(t) / d$, $P_{3}(t) / d$ are consecutive positive integers, with no prime factor greater than $t-\lambda$, where $\lambda=\min \left\{a_{i}: 1 \leqq i \leqq 6\right\}$. Hence $g(k, 3) \geqq P_{1}(t) / d$ for $k \geqq t-\lambda$.

For any integers $r, s$, the sequence $\left(b_{i}\right)_{i=1}^{6}$ also satisfies (13) and (14), where $b_{i}=r a_{i}+s$. Thus $b_{1}+b_{2}=0$ can be required, so the problem of solving (13) and (14) is equivalent to the problem of finding three squares $b_{1}^{2}, b_{3}^{2}, b_{5}^{2}$ which are in arithmetic progression. Dickson (1920) discusses this old problem; the solution which was known to Jordanus Nemorarius was published in 1496 . The general solution with $b_{1}>b_{3}>b_{5}>0$ is given by

$$
b_{1}=4 u+v+w, \quad b_{3}=2 u+v+w, \quad b_{s}=v-w,
$$

where $u, v, w$ are positive integers such that $v>w$ and $v w=2 u^{2}$. The smallest solution corresponds to $7^{2}, 5^{2}, 1^{2}$. By taking $r=2, s=7$, the corresponding 
solution to (13) and (14) leads to $P_{1}(x)=x(x+7), P_{2}(x)=(x+1)(x+6)$, $P_{3}(x)=(x+3)(x+4)$. As in (12), this gives $g(k, 3)>g(k, 9)^{2} / 6$ provided $k$ is large enough. Hence we have an effective proof of

THEOREM 4. For all sufficiently large integers $k$,

$$
g(k, 3)>\frac{1}{6} k^{20 / 9} .
$$

This construction does not extend to give a lower bound on $g(k, m)$ with $m \geqq 4$, for as Dickson (1920) reports Fermat knew, there do not exist four squares in arithmetic progression. However, such a lower bound (for $m \geqq 3$ ) could be given if we had a nontrivial integer sequence $\left(a_{i}\right)_{i=1}^{m n}$ such that

(16) $\sum_{i=1}^{n} a_{i}^{r}=\sum_{i=1}^{n} a_{j n+i}^{r} \quad$ for $\quad j=1,2, \cdots, m-1 \quad$ and $\quad r=1,2, \cdots, n-1$,

and

$$
\prod_{i=1}^{n} a_{(j-1) n+i}+\prod_{i=1}^{n} a_{(j+1) n+i}=2 \prod_{i=1}^{n} a_{j n+i} \quad \text { for } \quad j=1,2, \cdots, m-2 .
$$

We do not know whether such sequences exist; probably they do not.

\section{Bounds with explicit ranges of validity}

In this section we shall present numerical bounds for several inequalities in which the $g$ function exceeds some integral power of its first argument. The inequality (2) is an example of this type. The methods of proof are essentially adaptations of those used in $\$ 2$ and $\$ 3$; they are adequately illustrated in the proof of

\section{THEOREM 5. The inequality}

$$
g(k, 3)>k^{3}
$$

holds just if $k \geqq 41$.

PROOF. First we use cyclotomic polynomials to consider neighbours of a 30th power. By (3), all cyclotomic polynomials which are factors of $z^{60}-1$ are of degree 8 or less, except for $\Phi_{60}(z)$. But note the identity

$$
\Phi_{60}(z)=P^{2}(z)-2 z Q^{2}(z),
$$

where

$$
\begin{aligned}
& P(z)=z^{8}+z^{7}+2 z^{6}+3 z^{5}+3 z^{4}+3 z^{3}+2 z^{2}+z+1, \\
& Q(z)=z^{7}+z^{6}+2 z^{5}+2 z^{4}+2 z^{3}+2 z^{2}+z+1 .
\end{aligned}
$$


Thus when $2 z$ is a square, $\Phi_{60}(z)$ has a so-called Aurifeuillian factorization [cf. Cunningham and Woodall (1925)] as a difference of two squares, in which case each factor has leading term $z^{8}$. Indeed, if $z=2 t^{2}$ the three consecutive integers $z^{30}-1, z^{30}, z^{30}+1$ can have no prime factor greater than $P\left(2 t^{2}\right)+$ $2 t Q\left(2 t^{2}\right)$, which can be shown to be less than $365 t^{16}$ when $t \geqq 3$. For $365 t^{16} \leqq k<365(t+1)^{16}$ and $t \geqq 3$, it follows that $g(k, 3) \geqq g\left(365 t^{16}, 3\right) \geqq$ $2^{30} t^{60}-1>365^{3}(t+1)^{48}>k^{3}$. Thus (18) holds for $k \geqq q_{1}$, where $q_{1}=$ 11630180251 is the largest prime factor of $18^{60}-1$.

To show that (18) holds for smaller $k$, we now consider some specific factorizations of $z^{n}-1, z^{n}, z^{n}+1$, either with $z$ small, or with $n=6$. The largest prime factor of $2^{210}-1$ is $q_{2}=1564921$, so the three consecutive integers $2^{105}-1,2^{105}, 2^{105}+1$ show that (18) holds for $q_{2} \leqq k<2^{35}$. Since $q_{1}<2^{35}$, it follows that (18) holds for $k \geqq q_{2}$. Similarly, since the largest prime factors of $3^{78}-1,1073^{12}-1,695^{12}-1,12^{30}-1$ are respectively $q_{3}=797161, q_{4}=383419$, $q_{5}=241513, q_{6}=35671$, and $q_{2}<3^{13}, q_{3}<1073^{2}, q_{4}<695^{2}, q_{5}<12^{5}$, it follows that (18) holds for $k \geqq q_{6}$.

Next we apply the polynomials $P_{1}(x)=x(x+7), P_{2}(x)=(x+1)(x+6)$, $P_{3}(x)=(x+3)(x+4)$, constructed in $\$ 3$ from a solution of the generalized Prouhet-Tarry-Escott problem. By choosing $x$ so that the prime factors of $x$, $x+1, x+3, x+4, x+6, x+7$ are all suitably small and also $x \neq 1(\bmod 3)$, the triple of consecutive integers $P_{i}(n) / 6, i=1,2,3$ will afford an example for which (18) holds. In particular, with $x=16533113$ the corresponding three consecutive integers all exceed $35715^{3}$ (so each exceeds $q_{6}^{3}$ ) but their largest prime factor is only $q_{7}=1669$. Again, with $x=198912$ all three integers exceed $1875^{3}$ (so each exceeds $q_{7}^{3}$ ) and $q_{\mathrm{8}}=257$ is their largest prime factor. Similarly, the three consecutive integers corresponding to $x=3477$ exceed $126^{3}$, and $q_{10}=67$ is their largest prime factor.

The gap between 126 and $q_{8}$ can also be covered by several triples located in this way. However, we did not find a single triple which would suffice, so we considered triples located by evaluating the polynomials

$$
Q_{1}(x)=(x-1)(x+2), \quad Q_{2}(x)=x^{2}+x-1, \quad Q_{3}(x)=x(x+1) .
$$

In particular, with $x=6665$ all three integers exceed $354^{3}$ (so each exceeds $q_{8}^{3}$ ) and $q_{9}=113$ is their largest prime factor. Since $q_{9}<126,(18)$ holds for $k \geqq q_{10}$.

A direct search for triples located the examples whose smallest members are 302498 and 247455 . The largest prime factor on the first triple is $q_{11}=61$, and all three members exceed $67^{3}$ (so exceed $q_{10}^{3}$ ); the largest prime factor of the second triple is $q_{12}=53$, and all its members exceed $62^{3}$ (so exceed $q_{11}^{3}$ ). The results of Lehmer (1965), reproduced by Ecklund and Eggleton (1972), show $g(41,3)=212380$, so the members of the triple all exceed $59^{3}$ (so exceed $q_{12}^{3}$ ), 
and the largest prime factor present is $q_{13}=41$. Thus (18) holds for $k \geqq 41$. Lehmer's results for $g(k, 3)$ with $k<41$ show that (18) fails to hold in all cases, so the proof is complete.

A similar self-contained argument is possible when the $k^{3}$ in (18) is replaced by $k^{2}$. However, it is more convenient to deduce the result as a corollary of Theorem 5 (in conjunction with Lehmer's results):

$$
g(k, 3)>k^{2} \text { just if } k \geqq 13 \text {. }
$$

The corresponding inequality with $k^{4}$ as lower bound will now be considered. In this case we take neighbours of a 210 th power. All cyclotomic polynomials which are factors of $z^{420}-1$ are of degree 48 or less, except for $\Phi_{420}(z)$. Analogous to (19) is the identity

$$
\Phi_{420}(z)=R^{2}(z)-2 z S^{2}(z),
$$

where $R(z)$ and $S(z)$ are reciprocal polynomials (for example, $z^{48} R\left(z^{-1}\right)=$ $R(z))$, so are fully specified by

$$
\begin{aligned}
R(z)= & z^{48}+z^{47}+z^{46}+2 z^{45}-z^{43}-z^{42}+z^{40}+z^{39}+2 z^{38}-z^{36} \\
& -z^{35}-z^{34}-z^{32}+3 z^{31}-2 z^{29}-3 z^{28}-2 z^{27}-z^{26}+3 z^{24}-\cdots, \\
S(z)= & z^{47}+z^{46}+z^{45}-z^{41}+z^{39}+z^{38}+z^{37}-z^{35}-2 z^{34}+z^{32} \\
& +z^{31}-z^{28}-3 z^{27}-z^{26}+z^{24}+\cdots
\end{aligned}
$$

Thus, when $z=2 t^{2}$ there is an Aurifeuillian factorization of $\Phi_{420}(z)$, and the three consecutive integers $z^{210}-1, z^{210}, z^{210}+1$ can have no prime factor greater than $R\left(2 t^{2}\right)+2 t S\left(2 t^{2}\right)$, which is less than $2^{49} t^{\circ}$. For

$$
2^{49} t^{96} \leqq k<2^{49}(t+1)^{96}
$$

and $t \geqq 6$, we have $g(k, 3) \geqq 2^{210} t^{420}-1>k^{4}$. Moreover $2^{49} 6^{96}<3 \times 10^{89}$, so

$$
g(k, 3)>k^{4} \quad \text { holds if } \quad k \geqq 3 \times 10^{89} .
$$

The three consecutive integers $P_{i}(198912) / 6, i=1,2,3$, used in the proof of Theorem 5, provide an example showing that $g(k, 3)>k^{4}$ holds for $257 \leqq k \leqq$ 284 . This suggests that the bound $k^{4}$ in (22) may actually hold for $k \geqq 257$, and perhaps also for smaller values of $k$.

Clearly a result similar to (22) could be obtained for the bound $k^{5}$. The triple $2^{105}-1,2^{105}, 2^{105}+1$ used in the proof of Theorem 5 shows that $g(k, 3)>k^{5}$ holds for $1564921 \leqq k<2^{21}$.

It has been remarked by several authors that if the consecutive integers $a-1, a, a+1$ have no prime factors greater than $k$, then $a^{2}-1, a^{2}$ are consecutive integers with the same property. Hence if $g(k, 3)>k^{n}$ then 
$g(k, 2)>k^{2 n}$. In particular, Lehmer's results for $g(k, 2)$ can be combined with (18) to give

$$
g(k, 2)>k^{6} \quad \text { just if } \quad k \geqq 31 \text {, }
$$

and hence

$$
g(k, 2)>k^{5} \quad \text { just if } 19 \leqq k \leqq 25 \text { or } k \geqq 29 .
$$

Similarly, with (20) we have

$$
g(k, 2)>k^{4} \quad \text { just if } 7 \leqq k \leqq 8 \text { or } k \geqq 13 \text {, }
$$

and hence

$$
g(k, 2)>k^{3} \quad \text { just if } k \geqq 7 .
$$

We shall now derive a result for $g(k, 5)$ which is comparable to (22). All cyclotomic polynomials which are factors of $z^{44}-1$ are of degree 10 or less, apart from $\Phi_{44}(z)$, for which we have the identity

$$
\Phi_{44}(z)=T^{2}(z)-2 z U^{2}(z),
$$

where

$$
\begin{aligned}
& T(z)=z^{10}+z^{9}-z^{8}-z^{7}+z^{6}+z^{5}+z^{4}-z^{3}-z^{2}+z+1, \\
& U(z)=z^{9}-z^{7}+z^{5}+z^{4}-z^{2}+1 .
\end{aligned}
$$

Thus when $z=2 t^{2}, \Phi_{44}(z)$ has an Aurifeuillian factorization, and $z^{22}-1, z^{22}+1$ are necessarily greater than the squares of their largest prime factors if $t \geqq 2$ : the largest prime factors must be less than $1024 t^{19}(t+2)$.

Again, all cyclotomic polynomials which are factors of $w^{210}-1$ are of degree 48 or less, so if $w$ is an integer greater than $1, w^{105}-1, w^{105}+1$ are necessarily greater than the squares of their largest prime factors, which must be less than $w^{47}(w+2)$.

To combine these two observations, each of the consecutive integers $2\left(2^{n-1}-1\right), 2^{n}-1,2^{n}, 2^{n}+1,2\left(2^{n-1}+1\right)$ exceeds the square of its largest prime factor if (i) $n \equiv 22(\bmod 44)$ and $n \equiv 1(\bmod 105)$, or (ii) $n \equiv 23(\bmod 44)$ and $n \equiv 0(\bmod 105)$. Thus $n=4620 m+946$ or $n=4620 m+3675$, with $m \geqq 0$. In any case, the largest prime factor present cannot exceed $c .2^{16 n / 35}$, where $c=65 / 64$. Thus if $c .2^{2112 m+432} \leqq k<c .2^{2112 m+1680}$ and $m \geqq 7$, we have $g(k, 5) \geqq$ $2^{4620 m+946}-2>c^{2} .2^{4224 m+3360}>k^{2}$. Similarly, if $c .2^{2112 m+1680} \leqq k<c .2^{2112(m+1)+432}$ and $m \geqq 4$, we have $g(k, 5)>k^{2}$. These two results mesh for $m \geqq 6$; moreover c. $2^{14352}<10^{4321}$, so

$$
g(k, 5)>k^{2} \quad \text { holds if } k \geqq 10^{4321} .
$$


Evidently corresponding results could be established for higher powers of $k$ and also for $g(k, 4)$. However, the bounds obtained by this method are presumably far from best possible, so we content ourselves with the above illustration of the method. Note that the quintuple $2\left(2^{24}-1\right), 2^{25}-1,2^{25}, 2^{25}+1$, $2\left(2^{24}+1\right)$ shows that $g(k, 5)>k^{2}$ holds for $4051 \leqq k \leqq 5792$.

We shall close with some numerical information best presented in tabular form. For any positive integer $x$ and $m \geqq 2$, let $p(x, m)$ denote the largest prime factor of the $m$ consecutive integers $x+i, 0 \leqq i \leqq m-1$. Also let $a_{m}$ be the smallest positive integer such that $p\left(a_{m}, m\right)<\sqrt{a_{m}}$. In Table 1 we give the values of $a_{m}$ for $2 \leqq m \leqq 8$.

TABLE 1. Smallest $m$-tuples satisfying $g(k, m)>k^{2}$.

\begin{tabular}{rrrrrr}
\hline$a_{m}$ & $m$ & $p\left(a_{m}, m\right)$ & \multicolumn{1}{c}{$a_{m}$} & $m$ & $p\left(a_{m}, m\right)$ \\
\hline 80 & 2 & 5 & 28032 & 6 & 163 \\
350 & 3 & 13 & 290783 & 7 & 523 \\
1518 & 4 & 31 & 290783 & 8 & 523 \\
5828 & 5 & 67 & $>500000$ & 9 & - \\
\hline
\end{tabular}

Let $K_{m}$ denote the smallest positive integer such that $g(k, m)>k^{2}$ holds for all $k \geqq K_{m}$. Then (2) and (20) imply $K_{2}=5, K_{3}=13$. The examples in Table 2 suggest that $K_{4} \leqq 43, K_{5} \leqq 101, K_{6} \leqq 163$ and $K_{7} \leqq 337$. Lehmer's results imply $K_{4} \geqq 43$, so it seems almost certain that $K_{4}=43$, and probable that $K_{5}=101$. (If $K_{4} \neq 43$ then $K_{4}>653$, by Table 2 .)

TABLE 2. Ranges of $k$ which satisfy $g(k, m)>k^{2}$.

\begin{tabular}{rrrr}
\hline$x$ & $m$ & $p(x, m)$ & {$[\sqrt{x}]$} \\
\hline 10878 & 4 & 43 & 104 \\
17574 & 5 & 101 & 132 \\
38624 & 5 & 107 & 196 \\
28032 & 6 & 163 & 167 \\
44250 & 6 & 167 & 210 \\
52323 & 6 & 211 & 228 \\
99381 & 6 & 229 & 315 \\
190990 & 6 & 269 & 437 \\
356207 & 7 & 337 & 596 \\
426851 & 7 & 359 & 653 \\
\hline
\end{tabular}




\section{References}

A. J. C. Cunningham and H. J. Woodall (1925), Factorisation of $\left(y^{n} \mp 1\right)$, (Francis Hodgson, London, 1925).

L. E. Dickson (1920), History of the theory of numbers, Vol. 2 (Carnegie Institution, 1920).

E. F. Ecklund, Jr., and R. B. Eggleton (1972) 'Prime factors of consecutive integers', Amer. Math. Monthly 79, 1082-1089.

E. F. Ecklund, Jr., R. B. Eggleton and J. L. Selfridge (1973), 'Consecutive integers all of whose prime factors belong to a given set', Proc. Third Manitoba Conf. on Numerical Math., Univ. of Manitoba, Winnipeg, Oct. 3-6, 1973, pp. 161-162.

P. Erdös (1955), 'On consecutive integers', Nieuw Arch. Wisk. 3, 124-128.

G. H. Hardy and E. M. Wright (1960), An introduction to the theory of numbers (Clarendon, Oxford, 4th edn., 1960).

D. H. Lehmer (1964), 'On a problem of Størmer', Mlinois J. Math. 8, 57-79.

D. H. Lehmer (1965), 'The prime factors of consecutive integers', Amer. Math. Monthly 72, no. 2, part II, 19-20.

G. Størmer (1897), 'Quelques théorèmes sur l'equation de Pell $x^{2}-D y^{2}= \pm 1$ et leurs applications,' Videnskabs-Selskabets Skrifter, Christiania, 1897, no. 2, 48 pp.

\section{Department of Mathematical Sciences \\ Northern Illinois University \\ DeKalb, Illinois 60115, U.S.A.}

